\title{
Awareness of the New Kidney Allocation System among United States Dialysis Providers with Low Waitlisting
}

\author{
Rachel E. Patzer ${ }^{\mathrm{a}, \mathrm{b}}$ Mohua Basu ${ }^{\mathrm{a}}$ Kayla D. Smith ${ }^{\mathrm{a}}$ Laura Plantinga ${ }^{\mathrm{c}}$ \\ Sumit Mohan $^{d}$ Cam Escoffery $^{\text {e Joyce J. Kim }}{ }^{a}$ Taylor Melanson $^{f}$ \\ Stephen O. Pastan ${ }^{c}$ \\ ${ }^{a}$ Department of Surgery, Division of Transplantation, Emory University School of Medicine, Atlanta, GA, USA; \\ ${ }^{b}$ Department of Epidemiology, Rollins School of Public Health, Atlanta, GA, USA; ' Department of Medicine, Division

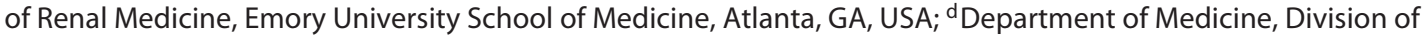 \\ Nephrology, College of Physicians and Surgeons, and Department of Epidemiology, Mailman School of Public \\ Health, Columbia University, New York, NY, USA; ${ }^{e}$ Department of Behavioral Sciences and Health Education, Rollins \\ School of Public Health, Atlanta, GA, USA; ${ }^{f}$ Department of Health Policy Management, Rollins School of Public \\ Health, Atlanta, GA, USA
}

\section{Keywords}

Kidney allocation policy · Provider knowledge · Kidney transplantation · Racial disparities

\footnotetext{
Abstract

It is unknown whether dialysis facility staff are aware of the new kidney allocation system implemented in December 2014, which changed how deceased donor kidneys are allocated and waiting time is calculated. U.S. dialysis facilities with low annual waitlisting $(<15.2 \%)$ were surveyed as part of a large randomized study. Among 653 facilities, $57.9 \%$ of staff were aware of the policy change, with medical directors (84.4\%) being more aware than social workers $(73.3 \%)$, facility administrators (53.1\%), nurse managers (46.4\%), and other staff (43.8\%). Targeted education among dialysis facilities with low waitlisting may help extend the reach of the new policy.

(c) 2018 S. Karger AG, Basel
}

In December 2014, the United Network for Organ Sharing implemented a new national kidney allocation system (KAS). While racial disparities were not the primary impetus, KAS changed how waiting time was calculated by starting the "clock" at the time of ESRD diagnosis, rather than when a patient is approved for waitlisting on the national deceased donor waiting list. Because of persistent barriers to accessing multiple steps of the kidney transplant process, black end-stage renal disease (ESRD) patients wait nearly twice as long for a deceased donor transplant compared to whites [1]. While preliminary studies of the effect of KAS found that black vs. white racial disparities in kidney transplantation were temporarily eliminated $[2,3]$, data suggest this effect may not be sustained [1]. To ensure that disparity reduction continues, it is essential for dialysis staff to continue to educate and refer patients for transplantation, particularly minority patients and those who have been on dialysis for sev-

\section{KARGER}

(c) 2018 S. Karger AG, Basel

E-Mail karger@karger.com

www.karger.com/ajn
Rachel E. Patzer, PhD, MPH

Department of Surgery, Division of Transplantation

Emory University School of Medicine, 101 Woodruff Circle

Woodruff Memorial Building \#5034, Atlanta, GA 30322 (USA)

E-Mail rpatzer@emory.edu 
eral years and could benefit from the KAS policy change by going to the top of the waiting list. However, the extent to which dialysis staff members are aware of KAS and its potential impact on patients' access to kidney transplantation is unknown. The purpose of the current analysis is to determine what proportions of providers at low-waitlisting dialysis facilities are aware of the recent KAS change. These results could illuminate whether more national campaigns aimed at KAS awareness are needed to improve equitable access to kidney transplantation.

With support from all 18 ESRD Networks in the United States, 1,529 facilities with low waitlisting $(<15.2 \%$ patients in facility waitlisted = lowest national tertile for waitlisting in 2014), >10 total patients, and $>3$ black patients were invited to participate in the Allocation System Changes for Equity in KidNey Transplant (ASCENT) study [4] in 2016. The goal of the larger randomized ASCENT study was to disseminate educational materials about kidney transplantation and the new KAS to both patients and staff at dialysis facilities with low waitlisting. At baseline, dialysis facilities received a link to an informed consent and online survey from their respective ESRD networks. Medical directors were asked to either complete the survey or forward to the staff member primarily responsible for transplant education at the facility, such as the nurse manager or social worker. This study focuses on a subset of data from the baseline ASCENT survey. The study protocol was approved by the Emory Institutional Review Board.

The ASCENT baseline survey was developed by a multidisciplinary group of dialysis facility medical directors and staff, ESRD networks, patients, researchers, and national advocacy groups [4]. The primary measure of interest was KAS awareness ("Were you aware the national KAS changed in 2014?"); responses were dichotomized into 2 groups: "Yes" vs. "No" or "Unsure". KAS/transplant knowledge was measured using a 5 -item scale, with 1 point for each correct item (online suppl. Table 1; for all online suppl. material, see www. karger.com/doi/10.1159/000486648). Questions about provider referral practices were also asked. Survey data were linked to 2014 data from Dialysis Facility Compare and United States Renal Data System to obtain dialysis facility characteristics. Descriptive and chi-square analyses were conducted using SAS 9.4 to explore differences in KAS awareness by provider and facility characteristics.

Among 653 providers from unique facilities, the majority were white $(60.6 \%)$, female gender $(73.3 \%)$, and worked at the facility for $1-5$ years $(48.2 \%$; Table 1$)$. A total of $17.6 \%$ of respondents were medical directors, 48.9\% nurse managers, $17.3 \%$ facility administrators, $13.8 \%$ social workers, and $2.4 \%$ other staff. Most facilities were for-profit (90.4\%) and located in the South (69.2\%). On average, these facilities treated 85 patients and the average proportion of patients who had been waitlisted in 2014 was $10.2 \pm 3.7 \%$. The mean age of the patient population was $61 \pm 4$, and about one-third of them were black.

Slightly over half (57.9\%) of facility staff were aware of the KAS policy change. Awareness was highest among medical directors (84.4\%) followed by social workers (73.3\%), facility administrators (53.1\%), nurse managers (46.4\%), and other staff (43.8\%; Table 1). Among those aware of KAS, staff reported hearing about KAS changes from a variety of sources, including transplant center outreach (42.1\%), their ESRD Network (40.5\%), or colleagues (26.2\%). Provider knowledge of KAS was significantly higher among those who were aware vs. unaware of the policy change (mean knowledge score $=2.96 \pm 1.30$ vs. $1.57 \pm 1.19 ; p<0.001$ ), and among medical directors (3.17 \pm 1.34$)$ followed by social workers $(2.69 \pm 1.40)$, facility administrators $(2.14 \pm 1.35)$, nurse managers $(2.10 \pm 1.38)$, and other staff $(2.06 \pm 1.44$; online suppl. Table 1). Clinical providers employed at the facility for $\geq 10$ years vs. $<1$ year ( 68.8 vs. $38.2 \%, p=<0.001)$ and providers from nonprofit vs. for-profit facilities (68.3 vs. $56.8 \%$; $p=0.08)$ were more aware of KAS. KAS/transplant knowledge was also higher among providers from non-profit vs. for-profit facilities $(2.81 \pm 1.42$ vs. $2.33 \pm$ $1.43 ; p=0.01)$. In addition, staff aware vs. unaware of KAS were more likely to report referring more patients overall ( 17.7 vs. $11.6 \% ; p=<0.001$ ), and referring more patients with $4+$ years on dialysis (12.7 vs. $4.0 \%$; $p=$ $<0.001$ ) since KAS implementation (Table 1). However, after adjustment for knowledge score, we found that these associations were no longer statistically significant among those who reported referring more patients overall $(\mathrm{OR}=1.48 ; 95 \% \mathrm{CI} 0.89-2.48)$, but still significant for providers who reported referring patients with $>4$ years on dialysis $(\mathrm{OR}=2.29 ; 95 \% \mathrm{CI} 1.10-1.70)$. There was no significant difference in KAS/transplant knowledge between providers who reported referring more patients overall, and those who did not report referring more patients overall ( 2.61 vs $2.33 ; p=0.08$ ). However, providers who reported referring more patients with $>4$ years on dialysis after the KAS change had significantly higher KAS/transplant knowledge scores than those who did not report referring more patients $(3.14$ vs 1.42 ; $p<$ $0.0001)$. In addition, we examined the percentage of pa- 
Table 1. Dialysis facility provider and facility characteristics associated with provider awareness of the new KAS

\begin{tabular}{|c|c|c|c|c|}
\hline \multicolumn{5}{|l|}{ Provider characteristics } \\
\hline Gender, $n(\%)$ & & & & $<0.001$ \\
\hline Male & 141 & $106(75.2)$ & $35(24.8)$ & \\
\hline Female & 479 & $252(52.6)$ & $227(47.4)$ & \\
\hline White & 396 & $230(58.5)$ & $163(41.5)$ & \\
\hline Black & 80 & $42(52.5)$ & $38(47.5)$ & \\
\hline Hispanic & 40 & $22(55.0)$ & $18(45.0)$ & \\
\hline Asian & 55 & $38(69.1)$ & $17(30.9)$ & \\
\hline Other & 21 & $9(42.9)$ & $12(57.1)$ & \\
\hline Missing* & 61 & $34(55.7)$ & $27(44.3)$ & \\
\hline Social worker & 90 & $66(73.3)$ & $24(26.7)$ & \\
\hline Other staff & 16 & $7(43.8)$ & $9(56.3)$ & \\
\hline Length of time at facility, $n(\%)$ & & & & $<0.001$ \\
\hline$<1$ year & 102 & $39(38.2)$ & $63(61.8)$ & \\
\hline $1-5$ years & 315 & $187(59.4)$ & $128(40.6)$ & \\
\hline $5-10$ years & 114 & $68(59.7)$ & $46(40.4)$ & \\
\hline$\geq 10$ years & 122 & $84(68.8)$ & $38(31.2)$ & \\
\hline KAS/transplant knowledge score (range: $0-5$ ), mean (SD) & $2.38(1.43)$ & $2.96(1.30)$ & $1.57(1.19)$ & $<0.001$ \\
\hline \multicolumn{5}{|l|}{ Reported referring more patients overall since } \\
\hline implementation of new KAS, $n(\%)$ & 99 & $67(17.7)$ & $32(11.6)$ & $<0.001$ \\
\hline \multicolumn{5}{|l|}{ Reported referring more patients with $>4$ years } \\
\hline dialysis since implementation of new KAS, $n(\%)$ & 59 & $48(12.7)$ & $11(4.0)$ & $<0.001$ \\
\hline \multicolumn{5}{|l|}{ Facility characteristics } \\
\hline Midwest & 120 & $69(57.5)$ & $51(42.5)$ & \\
\hline West & 54 & $26(48.1)$ & $28(51.9)$ & \\
\hline Number of staff in facility, mean (SD) & $16.3(10.3)$ & $16.5(11.3)$ & $16.1(8.7)$ & 0.60 \\
\hline Number of patients in facility, mean (SD) & $84.8(55.8)$ & $84.8(56.3)$ & $84.8(55.1)$ & 0.99 \\
\hline Number of black patients in facility, mean (SD) & $32.7(31.4)$ & $32.8(32.0)$ & $32.7(30.6)$ & 0.95 \\
\hline Percentage of patients waitlisted, mean (SD) & $10.2(3.7)$ & $10.3(3.6)$ & $10.2(4.0)$ & 0.82 \\
\hline Racial disparity in waitlisting, $n(\%)^{* * *}$ & 169 & $102(27.0)$ & $67(24.4)$ & 0.45 \\
\hline Age of patients in facility, mean (SD) & $60.6(4.1)$ & $60.7(4.0)$ & $60.5(4.2)$ & 0.69 \\
\hline
\end{tabular}

* Missing values not included in calculation of $p$ values.

** Northeast, CT, ME, MS, NH, RI, VT, NJ, NY, PA; South, DE, DC, FL, GA, MD, NC, SC, VA, WV, AL, KY, MS TN, AR, LA, OK, TX; Midwest, IL, IN, MI, OH, WI, IA, KS, MN, MO, NE, ND, SD; West, AZ, CO, ID, MT, NV, NM, UT, WY, AK, CA, HI, OR, WA, PR.

*** Facility considered to have racial disparity if \%whites waitlisted - \%blacks waitlisted $\geq 5 \%$. 
tients waitlisted by both years of staff experience and dialysis facility profit status and found no association between these variables, even when stratified by KAS awareness.

We found that $84.4 \%$ of medical directors and $73.3 \%$ of social workers in U.S. dialysis facilities with low waitlisting were aware of the new KAS policy change, but awareness was substantially lower among nurse managers, facility administrators, and other staff involved in transplant education. This suggests that, while knowledge of transplantation and KAS is high among medical directors, it may not be disseminated to the other staff who may serve as transplant educators within the facility. Results are consistent with other studies that have documented limited transplant knowledge among dialysis staff [5]. Fewer than $20 \%$ of dialysis staff report having detailed discussions about transplantation with their patients [5], and black patients are less likely to be educated about transplant as a treatment option [6]. In turn, limited staff knowledge about transplantation is associated with decreased patient access to transplantation [7], suggesting that staff education, and particularly culturally sensitive interventions, may be needed. While several educational interventions target dialysis staff, none have explicitly focused on KAS and how KAS could help some patient populations - such as minority and transplanteligible patients with long dialysis vintage - improve transplant access. Our findings that KAS/transplant knowledge was correlated with higher self-reported referrals for patients on dialysis $>4$ years - those expected to go to the top of the waiting list if transplant-eligible - suggest that more KAS education to dialysis facility staff could result in increased referrals for kidney transplantation. Our findings also suggest that targeting providers with lower knowledge and awareness of KAS (e.g., those with $<1$ year experience at a facility or providers at forprofit dialysis centers) with education regarding KAS and transplant in general may be beneficial and is consistent with previous literature [8].

This study has several limitations. Our sample is limited to dialysis facilities in the lowest national tertile of waitlisting, so results may not be generalizable to all facilities; however, these facilities were targeted in the larger study because these are facilities that could benefit most from the new policy. In addition, we did not find any association between KAS awareness and facility waitlisting, perhaps due to having only low-waitlisting facilities in the current study and a smaller variance in waitlisting; associations between facility waitlisting and KAS awareness would be interesting to explore among all U.S. dialysis facilities, regardless of levels of waitlisting. Another limitation is that our measures, including awareness of KAS, are self-reported so could be subject to misclassification and estimates may be biased upwards. However, KAS/transplant knowledge was associated with KAS awareness.

Results suggest that among dialysis facilities with low waitlisting, education campaigns targeted to dialysis staff, not just medical directors, may be needed. Ensuring that staff are aware of how they could help to extend the reach of KAS by referring potentially eligible patients who have been on dialysis for several years, including a disproportionate number of minorities, may help to further reduce disparities in access to kidney transplantation.

\section{Acknowledgments}

We would like to thank the National Institute on Minority Health and Health Disparities for funding this research (Grant Number 5R01MD010290-03).

\section{Disclosure Statement}

S.O.P. is a minority shareholder in Fresenius College Park Dialysis, in College Park, GA, USA. All other authors have no conflicts of interest. Some of the data reported were supplied by the United States Renal Data System. Interpretation and reporting of these data are the authors' responsibility and should not be seen as official policy or interpretation of the U.S. government.

\section{Funding Source}

National Institute on Minority Health and Health Disparities (grant number: R01MD010290). ClinicalTrials.gov number: NCT02879812.

\section{References}

1 United States Renal Data System: 2016 Annual Data Report: Epidemiology of Kidney Disease in the United States. Bethesda, MD, National Institutes of Health, National Institute of Diabetes and Digestive and Kidney Diseases, 2016.

2 Stewart DE, Kucheryavaya AY, Klassen DK, Turgeon NA, Formica RN, Aeder MI: Changes in deceased donor kidney transplantation one year after KAS implementation. Am J Transplant 2016;16:1834-1847.

3 Melanson TA, Hockenberry JM, Plantinga L, et al: New kidney allocation system associated with increased rates of transplants among black and hispanic patients. Health Aff (Millwood) 2017;36:1078-1085. 
4 Patzer RE, Smith K, Basu M, Gander J, Mohan S, Escoffery C, Plantinga L, Melanson T, Kalloo S, Green G, Berlin A, Renville G, Browne T, Turgeon N, Caponi S, Zhang R, Pastan S: The ASCENT (Allocation System Changes for Equity in Kidney Transplantation) Study: a randomized effectiveness-implementation study to improve kidney transplant waitlisting and reduce racial disparity. Kidney Int Rep 2017;2:433-441.
5 Waterman AD, Peipert JD, Goalby CJ, Dinkel KM, Xiao H, Lentine KL: Assessing transplant education practices in dialysis centers: comparing educator reported and medicare data. Clin J Am Soc Nephrol 2015;10:16171625.

6 Waterman AD, Peipert JD, Hyland SS, McCabe MS, Schenk EA, Liu J: Modifiable patient characteristics and racial disparities in evaluation completion and living donor transplant. Clin J Am Soc Nephrol 2013;8: 995-1002.

7 Gander J, Browne T, Plantinga L, et al: Dialysis facility transplant philosophy and access to kidney transplantation in the Southeast. Am J Nephrol 2015;41:504-511.

8 Balhara KS, Kucirka LM, Jaar BG, Segev DL: Disparities in provision of transplant education by profit status of the dialysis center. Am J Transplant 2012;12:3104-3110. 